\title{
Le passé historique dans les écritures québécoises du présent (Hamelin, Ouellette-Michalska, Leclerc, Mavrikakis)
}

\author{
O passado histórico nas escritas quebequenses do presente \\ (Hamelin, Ouellette-Michalska, Leclerc, Mavrikakis)
}

Robert Dion

Université du Québec à Montréal - Montréal - Québec - Canadá

\begin{abstract}
Résumé: Le présent article vise à examiner le statut du passé dans quatre romans québécois contemporains qui cherchent à l'inscrire dans le temps présent, ce qui les conduit à prendre leurs distances par rapport au sous-genre du roman historique - lequel ne met pas généralement l'accent sur un effort d'anamnèse engagé au présent, ni sur la quête obsessionnelle d'une "vérité du passé" capitale pour l'époque contemporaine. Après un premier survol des divers usages de l'histoire dans la prose narrative actuelle au Québec, il est possible de conclure qu'on y relève bel et bien des romans de l'histoire au présent, même si c'est souvent une histoire mineure, diffuse ou encore lointaine. Le fait que peu de romans prennent en charge les événements majeurs de la mémoire nationale ne veut certainement pas dire que le passé y est minoré; mais il semble qu'il soit encore difficile, pour la fiction québécoise, de revenir sur les épisodes clés de l'histoire pour les relier à des enjeux mémoriels, identitaires ou éthiques actuels.
\end{abstract}

Mots clés: Roman québécois; Histoire; Roman historique contemporain; Archives

Resumo: O presente artigo visa a examinar o estatuto do passado em quatro romances quebequenses contemporâneos que buscam inseri-lo no tempo presente, o que os leva a se distanciarem do subgênero do romance histórico - o qual geralmente não evidencia um esforço de anamnese engajada no presente, nem a busca obsessiva de uma "verdade do passado" capital para a contemporaneidade. Após uma primeira visada panorâmica sobre diversos usos da história na prosa narrativa atual no Quebec, é possível concluir que há sem dúvida romances da história no presente, mesmo que seja frequentemente uma história menor, difusa ou ainda distante. $\mathrm{O}$ fato de poucos romances tratarem de eventos maiores da memória nacional não quer dizer que o passado é subestimado; mas parece ser ainda difícil, para a ficção quebequense, retornar aos episódios-chave da história para relacioná-los a questões memoriais, identitárias ou éticas atuais.

Palavras-chave: Romance quebequense; História; Romance histórico contemporâneo; Arquivos

Il est devenu courant d'inscrire les littératures narratives contemporaines, en particulier celle de la France mais de plus en plus aussi celle du Québec, sous la bannière de la filiation et de l'héritage $^{1}$, et plus largement de l'histoire et de la mémoire. Pour plusieurs observateurs, critiques ou même historiens, tout semble

\footnotetext{
1 En témoignent entre autres, pour la France, l'ouvrage de Laurent Demanze, Encres orphelines. Pierre Bergounioux, Gérard Macé, Pierre Michon (2008), et le collectif publié sous la direction de Gianfranco Rubino, Présences du passé dans le roman français contemporain (2007); pour le Québec, le collectif récemment publié sous la direction de Karine Cellard et Martine-Emmanuelle Lapointe, Transmission et héritages de la littérature québécoise (2011).
}

en effet se passer comme si, à la suite du reflux des avant-gardes constaté à partir des années 1980, un verrou avait sauté en même temps que l'injonction de faire table rase du passé et de se tourner vers un avenir forcément radieux: depuis, une part importante de la littérature et des discours qui l'accompagnent serait devenue obsédée par le passé et par le souvenir - quand elle ne cherche pas, par une sorte de paradoxe, à patrimonialiser le présent, c'est-à-dire à y déceler déjà ce qui, un jour, sera digne d'être retenu, archivé (voir HARTOG, 2003). Il y a sans doute quelque exagération à cette lecture de la production littéraire actuelle comme réinvestigation têtue de 
l'histoire plus ou moins récente, à tout le moins en ce qui concerne le Québec. En fait, quand on regarde de plus près la littérature québécoise d'aujourd'hui, on y découvre relativement peu d'entreprises de relecture, depuis le point de vue du présent, d'événements historiques qui constitueraient des "nœuds" qu'il conviendrait de dénouer une fois pour toutes. Au contraire de ce que l'on observe en France, on ne trouve pas, dans ce corpus, de roman qui revienne sur les dérives des régimes fascistes des années 1930 et 1940 (par exemple, Didier Daeninckx, Meurtres pour mémoire [1984]), sur les séquelles d'une guerre sale (Laurent Mauvignier, Des hommes [2009]) ni sur les errements politiques de la jeunesse extrémiste des années 1960 (Olivier Rolin, Tigre en papier [2002]).

Ce n'est pas, cela dit, que le roman québécois fasse complètement l'impasse sur l'histoire: la vogue du roman historique - d'Arlette Cousture (les Filles de Caleb, tomes 1, 2 et 3 [1985, 1986 et 2003]) à Louis Caron (les Fils de la liberté, tomes 1, 2 et 3 [1981, 1982 et 1990]) en passant par Michel David ('̀ l'ombre du clocher, tomes 1, 2, 3 et 4 ([2006, 2007, 2007a, 2008]) et Micheline Lachance (le Roman de Julie Papineau, tomes 1 et 2 [1995 et 1998]) indique tout le contraire. Mais s'il traduit nécessairement les préoccupations du temps de l'écriture ${ }^{2}$ (l'anachronisme constituant d'ailleurs l'un des écueils où il risque sans cesse de s'échouer), le roman historique - qu'il soit "nouveau", visant alors "la représentation de toutes les identités (postcoloniales, migrantes, féminines, etc.) mises de l'avant à l'ère postmoderne" (DESBIENS, 2006, p. 28), ou qu'il s'inscrive dans le droit fil de la tradition inaugurée par Walter Scott - ne met généralement pas l'accent sur un effort d'anamnèse engagé au présent, ni sur la quête obsessionnelle d'une "vérité du passé" capitale pour l'époque contemporaine. Le savoir historique y est bien plutôt présupposé, constitué avant même que ne se déploie l'écriture ou la diégèse; il n'est que très rarement problématisé, et ses sources - témoignages, archives, travaux historiques, etc. - ne sont à peu près jamais indiquées, sinon hors texte, et encore moins remises en question. Bref, nous manquons peut-être au Québec de ces romanciers qui s'apparentent à ce que Pierre Nora, cité par François Hartog, appelle des "historiens du présent" qui soient aptes à faire "consciemment surgir le passé dans le présent (au lieu de faire inconsciemment surgir le présent dans le passé)" (NORA, cité dans HARTOG, 2003, p. 136).

Il en va tout autrement en France au sein d'une production qui affiche son souci d'interroger l'histoire afin de dénouer certains blocages de la mémoire; comme le remarque Nathalie Piégay-Gros à propos des Géorgiques (1981) de Claude Simon, texte emblématique de ce retour au passé enclenché dans la décennie 1980, l'archive change ici de statut: matériau non pas préalable mais exposé dans sa matérialité sensible, poussiéreuse et fragile; incomplète par définition, elle [l'archive] ne promet aucune reconstitution parfaite de la réalité. Elle articule la mémoire à l'histoire, personnelle et collective, une mémoire incertaine, faite de tremblements, d'hypothèses et de doutes. (PIÉGAY-GROS, 2012, p. 28)

Il n'y a pas que l'archive qui soit ébranlée au sein de la production narrative contemporaine française; PiégayGros étend le constat à la mémoire elle-même:

Si la mémoire devient insistante, impérieuse même, s'il faut se rappeler, commémorer, pour savoir qui l'on est, si l'histoire a pris le pas sur la littérature dans l'imaginaire collectif - et la migration des dispositifs historiques dans le texte littéraire ne cesse de le confirmer -, la remémoration est désormais consciente de sa fragilité, soucieuse de montrer les traces qui la fondent, inquiète de leurs implicites, de leurs impostures et trucages possibles. (PIÉGAYGROS, 2012, p. 50)

Si donc le roman québécois actuel ne s'est pas systématiquement attaché à lever les tabous qui pèsent sur la mémoire - à la manière des récits sur l'Occupation et la guerre d'Algérie en France, sur le nazisme en Allemagne ou sur l'esclavagisme et la guerre du Vietnam aux États-Unis -, il n'est toutefois pas resté indifférent aux soubresauts de l'histoire. Chose curieuse, il se signale entre autres par le fait, somme toute inusité, que c'est souvent l'histoire des autres qui a suscité ses interrogations (on songe au Ciel de Bay City de Catherine Mavrikakis [[2008] 2011], mais aussi à l'Homme blanc de Perrine Leblanc [2010], à Revoir Nevers de Roger Magini [2006], à Du bon usage des étoiles de Dominique Fortier [2008] $)^{3}$, comme si les romanciers du Québec avaient du mal à relier l'expérience de leur passé propre à leur situation présente, à en extrapoler les répercussions dans un hic et nunc devenu par là même mieux interprétable. Loin de pallier cette lacune, la vogue du roman historique traditionnel à la Micheline Lachance témoignerait à l'opposé de la forclusion d'un passé certes national mais enfermé sur lui-même, sans conséquences sur le présent - d'un passé à la fois exotisé par l'exhibition soigneuse de différences de surface et normalisé par la projection implicite de schèmes idéologiques contemporains

\footnotetext{
2 C'est une affaire généralement entendue que le roman historique représente "non pas une évasion dans le passé mais une explication [du] présent, une vision de [l']avenir" (DASPRE, 1975, p. 244).

3 Le roman de Mavrikakis met en scène une juive américaine obsédée par la Shoah, celui de Leblanc un enfant né au goulag, celui de Magini un journaliste qui se rend au Mexique pour écrire un article sur le soixantième anniversaire du bombardement d'Hiroshima; quant au roman de Fortier, il relate l'expédition désastreuse de sir John Franklin pour découvrir le passage du Nord-Ouest.
} 
sur des situations qui ne sauraient en aucun cas les admettre ${ }^{4}$.

Dans ce qui suit, je voudrais d'abord m'arrêter à un cas, celui de la Constellation du lynx de Louis Hamelin ([2010] 2012), qui s'apparente d'assez près aux romans de l'histoire français contemporains - il s'agit d'ailleurs de l'une des rares œuvres québécoises récentes qui "revisite" au présent un épisode traumatisant de l'histoire nationale ${ }^{5}$. Puis j'analyserai brièvement deux textes sans doute plus typiques de la manière québécoise d'intégrer la référence historique: aussi bien la Maison Trestler de Madeleine Ouellette-Michalska (1984) que la Patience des fantômes de Rachel Leclerc (2011) font appel à un substrat historique personnel, plus subjectif et plus diffus, ressortissant à l'ordre du familial, voire du féminin; dans les deux cas également, le projet de reconstitution historique est soumis à une commande idéologique qui le dépasse. Enfin, je terminerai en évoquant l'exemple à mon sens le plus frappant, dans le roman québécois récent, d'appropriation de l'histoire de l'Autre: le Ciel de Bay City, de Catherine Mavrikakis. J'espère ainsi parvenir à opérer un premier survol des divers usages de l'histoire dans la prose narrative actuelle au Québec, en écho à un mouvement contemporain plus général et plus large.

\section{La Constellation du Iynx}

Il serait présomptueux de faire de la Constellation du lynx $x^{6}$ le seul roman québécois publié depuis 1980 - ou le meilleur - qui rende compte d'une mémoire inquiète à l'égard d'un épisode historique québécois majeur (en l'occurrence, la crise d'Octobre 1970) ${ }^{7}$.

Publié tout juste 40 ans après la crise et participant de ce fait, volontairement ou non, de l'esprit commémoratif souvent relié au 'devoir de mémoire'8, la Constellation $d u$ lynx a d'abord frappé la critique par son ampleur 593 pages bien tassées dans l'édition de poche ${ }^{9}-$, par son ambition - huit ans de recherches soutenues pour parvenir

\footnotetext{
4 Selon l'historienne Micheline Dumont, ce serait le cas par exemple de la notion de couple dans Lady Cartier (2004) de Micheline Lachance. Personnage du XIX ${ }^{\mathrm{e}}$ siècle, "Lady Cartier ne peut pas se lamenter sur sa 'vie de couple', concept formulé à la fin du XX ${ }^{\mathrm{e}}$ siècle" (DUMONT, 2006, n. p.)

5 Il y a bien sûr aussi, parmi les œuvres un peu moins récentes, l'important Volkswagen Blues (1984) de Jacques Poulin, qui superpose à la traversée de l'ouest américain la lecture, par les protagonistes, des livres d'histoire qui s'y rapportent. J'ai déjà abordé ce roman ailleurs (DION, 2011).

6 Dorénavant, les références à cet ouvrage seront appelées par le sigle $C L$ et le folio, entre parenthèses.

7 Hormis Volkswagen Blues, qui accorde une attention toute particulière aux perdants et aux désillusionnés de la conquête du continent américain, on trouve par exemple le Bateau d'Hitler de Pierre Turgeon (1988), qui envisage les liens entre un certain nationalisme québécois des années 1930 et l'Allemagne nazie. Ce roman, toutefois, s'il part de certaines données historiques réelles, dévie rapidement vers une sorte de politiquefiction qui l'entraîne du côté de l'œuvre de pure imagination, voire du thriller. Sur les événements d'Octobre eux-mêmes, il y a aussi, toujours de Turgeon, Un dernier blues pour Octobre et, de Louis Caron, le troisième
}

à reconstituer la crise de tous les points de vue, autant celui des felquistes que celui des policiers, des militaires et du personnel politique, et depuis toutes les époques, de la fin de la Deuxième Guerre mondiale, alors que l'idée indépendantiste commence à germer, jusqu'aux années 2000, au moment où se sont déposées d'innombrables couches d'interprétations souvent divergentes - et par son parti pris nettement affirmé d'opposer une fiction romanesque aux fictions de l'histoire élaborées tant par les ravisseurs de James Richard Cross et de Pierre Laporte que par les autorités policières et politiques. À la journaliste Noémi Mercier qui lui demandait d'indiquer, au sein de son roman, la part du réel et de la fiction, Hamelin, usant d'une stratégie de brouillage, donnait la réponse suivante:

La crise d'Octobre est en soi un roman passionnant. Vous seriez étonnée de constater à quel point j'ai peu inventé. À mon humble avis, mon évocation est plus proche de la vérité que tout ce qui a été raconté jusqu'à maintenant. En même temps, je tiens à ce que l'ouvrage soit lu comme un roman. (HAMELIN, dans MERCIER, 2010, n. p.)

Le critique Michel Lapierre, pour sa part, se faisait le relais fidèle du romancier dans un article où il disait enfin comprendre que la crise d'Octobre "a tellement traumatisé notre conscience collective que seule la lunette de la fiction peut nous en faire découvrir la vérité cachée, l'essence qu'obscurciraient toute exactitude anecdotique, tout sensationnalisme lié à des noms véritables" (2010, n. p.).

En clair, malgré quelques rares voix discordantes - celles de protagonistes ou de témoins directs le plus souvent, dont les versions ne sont pourtant guère plus crédibles, aux yeux d'Hamelin, que les autres récits de la crise -, un fort consensus en faveur du caractère légitime du recours au roman pour élucider enfin les événements d'Octobre 1970 semble vouloir se dégager au sein de la critique. On a ainsi reconnu que l'auteur avait non

tome de la saga des Fils de la liberté, le Coup de poing, deux livres publiés en 1990 pour commémorer les 20 ans de la crise. Ces romans sont toutefois plus ou moins réussis. Pierre Hébert met bien le doigt sur le problème du Coup de poing quand il note que le récit, "[a]près un début relevant de la fiction proprement dite [...], par ses retours en arrière, récupère l'histoire d'Octobre 1970, souvent à la manière d'un reportage" (1991, p. 541), de telle sorte qu'à cause “de cette 'reproduction' de documents historiques, de ces métarécits et retours en arrière à fonction démonstrative ou explicative, à cause aussi des segments de reportage lancés dans le texte", le romancier n'a d'autre recours que d'installer une narration quasi-despotique qui "force pratiquement l'Histoire à entrer dans la fiction alors que la fiction ellemême n'est pas encore tout à fait tissée" (p. 542). Quant à Un dernier blues pour Octobre, il s'agit d'un roman historique plutôt conventionnel, qui transpose les événements réels dans un cadre très romanesque.

8 L'expression est apparue au début des années 1990, d'abord dans les journaux, à l'occasion de l'arrestation du milicien Paul Touvier et de son procès. Elle sera reprise ensuite à répétition dans les médias de masse, jusqu'à susciter l'intérêt des historiens et des philosophes: voir entre autres Ferenczi (2002)

9 Édition, je le rappelle, que j'utilise ici. 
seulement le droit de s'aventurer "dans des zones que les historiens officiels ne peuvent explorer, parce que leur travail repose essentiellement sur des archives écrites", mais qu'il avait eu raison de se servir de son imagination pour fouiller cette affaire, seul le roman pouvant lui permettre "d'ouvrir des pistes et d'embrasser l'histoire dans toutes ses contradictions, ses ambiguïtés, ses coins d'ombre" (HAMELIN, dans MERCIER, 2010, n.p.). Peut-être est-ce parce que la presse culturelle et politique a reconnu d'emblée la qualité littéraire de sa proposition ${ }^{10}$ qu'Hamelin s'est assez peu soucié d'en défendre les mérites esthétiques; toujours est-il que, si on l'a beaucoup vu dans les médias à la sortie du livre, c'est d'abord sur le terrain de la polémique historique qu'il est intervenu, ce qui a conduit à occulter la question, pourtant loin d'être secondaire, des vertus cognitives spécifiques de l'enquête romanesque et, accessoirement, de la valeur proprement littéraire et romanesque de la mise en récit et de la fictionnalisation des données de l'enquête historique.

La Constellation du lynx illustre tout à fait cette "migration des dispositifs historiques dans le texte littéraire" que Piégay-Gros affirme être caractéristique d'un nombre considérable d'œuvres contemporaines. C'est particulièrement le cas dans les passages qui mettent en scène les personnages de Chevalier Branlequeue - transposition approximative d'un Jacques Ferron mâtiné de Gaston Miron -, de Sam Nihilo - alter ego romanesque d'Hamelin -, de Frédéric Falardeau et des autres membres du cercle des "Octobierristes", composé d'étudiants de l'UQAM qui se réunissaient régulièrement autour de Branlequeue dans un bar de la rue Ontario pour élucubrer sans fin - leur mentor étant l'auteur immortel des Élucubrations! - sur les dessous de la crise d'Octobre. Dans ces passages, les thèses des uns et des autres sont passées au crible du soupçon conspirationniste. Branlequeue et ses disciples sont en effet convaincus - comme du reste Hamelin lui-même - que la version officielle selon laquelle la crise aurait pris les autorités au dépourvu est un grossier mensonge: les services de renseignement savaient que des actions d'éclat se tramaient, ils avaient infiltré le FLQ, incitant même les terroristes à agir; ils auraient sacrifié Pierre Laporte avec d'autant plus de cynisme que les liens de celui-ci avec la mafia, qui faisaient l'objet d'une enquête policière, menaçaient d'éclabousser le parti Libéral. Je n'entrerai pas plus avant dans la discussion de la thèse des Octobierristes, sinon pour ajouter que leur position, et par conséquent celle d'Hamelin, ne va pas jusqu'à attribuer aux forces de l'ordre la totale responsabilité de la fabrication de la crise à la façon de ces "truthers" américains pour qui le 11-Septembre est une machination de la CIA et du FBI. Ce que les personnages et l'auteur soutiennent en revanche, c'est que ce ne sont pas des circonstances imprévisibles ni une série de hasards malheureux qui ont abouti à la mort d'un homme, mais un enchevêtrement de forces complexes, d'intentions croisées ${ }^{11}$.

Cette complexité aux suites funestes, les personnages la mettent au jour au fil d'une enquête qui emprunte largement aux méthodes de l'histoire: compulsion et confrontation des récits historiques, des rapports et des témoignages, des coupures de presse, des minutes de procès et des pièces à conviction, des interviews (anciennes et récentes), etc. Tant et si bien que la crise apparaît après coup, dans l'optique résolument textuelle qui est celle des disciples de Branlequeue, comme résultant d'abord de l'entrechoquement violent de versions du réel contradictoires consignées dans les archives, versions qui, comme en conviennent Sam Nihilo et Fred Falardeau plusieurs années après les beaux jours du cercle octobierriste, s'apparentent peu ou prou à des fictions:

Avoue qu'on est loin de Joyce. On est loin d'Hubert Aquin.

Pas d'accord. On est dans l'invention et la fabrication, l'intrigue et l'histoire. Des gens très créatifs, là aussi, lancent leurs petites fictions dans le monde. La différence, c'est que quand ça fonctionne, on n'appelle pas ça un best-seller. On appelle ça l'Histoire...

Mais de quoi tu parles, là ?

De la désinformation comme un des beaux-arts. À une certaine profondeur, ce qu'on trouve, ce n'est plus des gens qui se tirent dessus, plutôt une guerre entre des textes. (CL, p. 346-347)

Décrits tels des "littéraire[s] égaré[s] [...] dans les souterrains de l'histoire secrète" (CL, p. 348), les deux protagonistes sont des professionnels de la fiction et des intrigues, de la mise en regard et en miroir des textes. Ils sont par conséquent convaincus du pouvoir de la fiction non seulement à dire le monde mais à le façonner. Ils seraient sans doute d'accord avec Michel de Certeau pour affirmer que le passé est "fiction du présent" ([1975] 2002, p. 24): une fiction qui s'élabore au présent afin de conférer un usage au passé. Selon Nihilo, la crise d'Octobre "était restée [...] la face cachée de la lune québécoise" ( $C L$, p. 510): en somme, une histoire que trop de textes contradictoires avaient rendue illisible, et pour ainsi dire impropre à servir, "hors d'usage". Peut-être serait-il plus juste de dire que cette histoire - c'est-à-dire l'Histoire ${ }^{12}$

\footnotetext{
${ }^{10}$ Avec parfois un accent d'enthousiasme frisant l'outrance: voir par exemple Bergeron (2010), qui parle du roman comme du "premier chef-d'œuvre romanesque occidental engendré par la culture québécoise" (n. p.), rien de moins!

11 Voir les propos d'Hamelin rapportés par Robitaille (2010).

12 Je reprends ici la majuscule à "Histoire" qui apparaissait dans le dialogue cité plus haut entre Sam Nihilo et Fred Falardeau.
} 
telle qu'il s'agirait idéalement de l'exhumer - avait été pratiquement recouverte par les fictions des uns et des autres. Au terme du roman, quand le fin mot de la crise apparaît à Sam Nihilo dans toute sa limpide clarté, c'est comme si les couches de récits tombaient une à une:

Toute une histoire. Mais leur couverture narrative les avait trahis, la petite histoire concoctée exprès pour tromper les journalistes et leur fournir une réponse toute prête aux questions des voisins [...]. Leur fabrication était posée comme un couvercle sur la vraie histoire, si prodigieusement secrète que mes paroles, en lui donnant forme, me causaient un choc répété, car tandis qu'elles sortaient de ma bouche, je découvrais que les choses avaient vraiment dû se passer ainsi. ( $C L$, p. 556)

En parallèle, l'enquête historique de Nihilo et l'enquête romanesque d'Hamelin finissent donc par produire une histoire officieuse "de rechange", qui zigzague entre les versions des autorités et celles des felquistes, et qui prétend déjouer les pièges de la désinformation. Par les moyens conjugués de la discipline historique et de la fiction - sauts dans l'espace-temps, colmatage des brèches du récit, polyphonie, variations de points de vue, focalisation interne, scènes, interpolations et extrapolations, etc. -, cette histoire autre débouche sur une fiction plus englobante que les fictions historiques existantes et, partant, plus puissante qu'elles. Elle présente aussi l'avantage de ruser avec l'une des fatalités de l'écriture historienne mises en lumière par de Certeau; celui-ci remarque en effet que la recherche "débute dans l'actualité d'un lien social et d'un appareil institutionnel ou conceptuel déterminés", alors que "l'exposé suit un ordre chronologique" dans la mesure où "[i]l prend le plus ancien comme point de départ" ([1975] 2002, p. 120) - ce qui constituerait une forme de distorsion quasi inévitable. Or, par sa construction extrêmement fragmentée, qui multiplie les points de vue sur la crise, la Constellation du lynx échappe à cette inversion de la perspective: si la quête de Sam Nihilo, qui a pour origine la décision de la succession Branlequeue de lui confier les papiers de l'écrivain défunt (nous sommes alors en 2000), se trouve à inscrire dans le roman les déterminations conceptuelles et idéologiques du présent et à rapatrier le passé dans ce présent, de nombreux autres plans de récit, sans lien direct avec ce niveau premier, se situent de plain-pied dans un passé qu'ils évoquent au présent, en parfaite contemporanéité avec l'événement. Le roman s'ouvre ainsi, de manière aussi surprenante que spectaculaire, à L'Avenir en 1975, par cette phrase: "Je m'appelle Marcel Duquet ${ }^{13}$ et je vais mourir dans environ cinq minutes" ( $C L$, p. 17). Cette narration impossible, en plus de produire un effet littéraire saisissant, ne trouve aucun prolongement dans le présent (normalement, le récit n'aurait même pas dû se rendre jusqu'à nous): elle instaure un passé clos, qui ne se relie à aucun présent parce qu'il est lui-même un présent qui ne peut s'abolir. Beaucoup de séquences de la Constellation du lynx, si elles ne sont pas à ce point frappantes, éclairent les prodromes de la crise d'Octobre sans les relier au projet de sens contemporain que poursuit Sam Nihilo. Le passé est ainsi donné pour ce qu'il est: un épisode enkysté dans le temps, inatteignable par le souvenir, non mobilisable pour l'édification du récit historique, mais doté d'une forte présence littéraire et romanesque, et qui résonne librement chez le lecteur. C'est dans cette relative déliaison du récit, qui fait alterner des plages de senspour-nous, pour notre présent, et des moments de passé restitués à leur propre chronotope, que réside, à mon sens, l'indéniable littérarité du roman d'Hamelin, laquelle tient au caractère complexe de son pouvoir de suggestion.

\section{Une histoire diffuse: la Maison Trestler et la Patience des fantômes}

Les petites histoires sont bien plus tenaces que la grande histoire, elles reviennent toujours comme un boomerang et impliquent des événements contre lesquels on ne peut pas grand-chose [...].

(RACHEL LECLERC, la Patience des fantômes, p. 248)

Le roman de Madeleine Ouellette-Michalska, la Maison Trestler ou le $8^{e}$ jour d'Amérique ${ }^{14}$, se donne d'emblée pour davantage qu'un roman historique. En quatrième de couverture, on lit en effet que "ce livre touchant, moderne, souvent drôle, fait plus que raconter une saga familiale", puisque "[1]'actualité politique, les rêves, les souvenirs de lectures que la narratrice faisait, enfant, dans le vieux grenier familial, font surgir des faits anciens et actuels qui interrogent le passé et l'avenir en regard de l'attachement porté à la France et de la dangereuse proximité américaine" ( $M T, 4^{\mathrm{e}}$ de couv.). Ce qui est décrit dans ces lignes, c'est en fait, grosso modo, le programme type des romans contemporains qui entendent revenir sur l'histoire pour en dégager "revenances" (HAMEL, 2006) et rémanences.

Alter ego de l'auteure, journaliste et écrivaine comme elle, la narratrice, dans la Maison Trestler - du nom du bâtiment historique qui constitue, littéralement, l'une de ces "maisons du Père" décrites par les critiques

\footnotetext{
${ }^{13}$ Alias Michel Viger, militant indépendantiste qui cacha les frères Rose et Francis Simard dans sa maison de Saint-Luc, près de Saint-Jean-surRichelieu, après l'exécution de Pierre Laporte. Il n'est pas mort assassiné comme le raconte Hamelin, mais écrasé par son tracteur.

${ }^{14}$ Dorénavant, les références à cet ouvrage seront appelées par le sigle $M T$ et le folio, entre parenthèses.
} 
féministes (voir SMART, 1988) -, entreprend de raconter, voire d'imaginer l'histoire de la fille de JeanJoseph Trestler, mercenaire allemand arrivé au Québec en 1776 et fondateur d'une prospère lignée. Catherine, cette enfant rebelle qui sera déshéritée par son père pour avoir épousé sans son consentement l'un de ses commis, fascine la narratrice qui s'y reconnaît et s'y projette jusqu'à fusionner avec elle dans un je parfois indistinct. Tout en racontant ce qu'on peut savoir de Catherine en dépit de la rareté des archives qui la concernent (et qui concernent les femmes en général), la narratrice relate ses visites à la Maison Trestler, un centre culturel fondé par ses hôtes Éva et Benjamin, plonge dans son propre passé, réfléchit à l'écriture de son roman comme à celle de l'histoire. Le texte se caractérise donc par la "variabilité de la focalisation avec ses mouvements continuels d'ouverture, de rétrécissement, de superposition des champs de vision" et par la "fluidité du temps qui, entremêlant les fils des deux histoires, fait passer le lecteur, dans le discontinu même de la rêverie, de l'époque de Trestler à celle de la narration, à près de deux siècles de distance" (GONTARD, 2003, p. 567). En cela, la Maison Trestler constitue bien un roman historique postmoderne tel que le décrivent Marie-Frédérique Desbiens (voir supra) et Janet M. Paterson, qui, dans Moments postmodernes dans le roman québécois, note justement à propos du roman de Ouellette-Michalska:

Penser à l'Histoire, se penser dans l'Histoire, repenser l'Histoire ou même se situer historiquement pour s'interroger comme sujet écrivant sont des constantes qui apparaissent dans plusieurs romans historiques postmodernes. (PATERSON, [1990] 1993, p. 54)

La critique de l'histoire passe ici par sa démonumentalisation et par ce qu'on pourrait appeler sa corrélative "diffusion". Il s'agit non seulement d'instruire le procès de l'histoire, mais d'en ébranler l'édifice en y introduisant des matériaux plus friables, plus fragiles; à l'histoire des vainqueurs (les Anglais contre les Français, les Français contre les "Indiens", les Britanniques contre les Canadiens français) et à l'histoire patriarcale figurée par Jean-Joseph Trestler, la narratrice oppose ainsi l'histoire occultée des femmes et de la sphère privée et domestique, quotidienne. Cette histoire ne saurait être qu'évanescente, diffuse, entremêlée au bavardage de la vie familière et en bonne partie non écrite: les femmes, en effet, ont peu écrit, et ce qu'elles ont écrit a été minoré, ou marginalisé, ou détruit. Au poids écrasant de l'histoire officielle et patriarcale répond donc, comme son envers, l'insoutenable légèreté de l'histoire des femmes, tout aussi intolérable. La narratrice se sent par conséquent investie de la mission d'écrire cette histoire, celle de Catherine comme celle d'Éva, et la sienne propre, de la seule manière possible: par bribes, faute de sources; par procuration, le destin de chaque femme consonant avec celui de toutes les autres; et en ayant recours à la fiction, dont la cohérence obligée est, selon Ouellette-Michalska, garante d'une certaine fidélité au réel, et qui permet d'explorer les voies depuis toujours négligées de l'écriture de l'histoire: histoire du corps, des sensations, des odeurs, etc. (par exemple: "Réveil des sens. Le récit s'organisera autour d'odeurs chaudes" - MT, p. 41).

La narratrice est bien consciente que c'est d'ellemême qu'il s'agit à travers cette écriture, cette quête et cette ressaisie d'un personnage qui appartient à une histoire refoulée:

Pourquoi ai-je moi-même souhaité percer le mystère de Catherine Trestler, celui de son père, d'Éva, de Benjamin, de Monsieur $\mathrm{B}^{15}$ ? Le mystère n'existe que d'être imaginé. Un roman de trois cent pages m'apprendra peut-être que nous sommes les visages d'une seule et même personne. Un être sans âge qui endosse, dans sa traversée de l'espace et du temps, un ensemble de vies et de morts lui apportant la plénitude d'existence qu'une seule vie et une seule mort ne sauraient satisfaire.

L'ombre de Catherine qui rôde dans cette pièce est plus que l'ombre de Catherine. Et cette cheminée de pierre renvoie à d'autres feux, d'autres pierres, d'autres drames, d'autres maisons. Cette nuit, j'entendais tousser Éva à travers mon sommeil, et j'avais la certitude que Benjamin n'était pas rentré, que Catherine avait déjà souffert d'une maladie respiratoire dans des circonstances analogues, que ma santé s'en trouvait affectée. Je tâte ma gorge. Des ganglions roulent sous mes doigts. À mon retour, Stefan dira que je commence la dix-neuvième grippe de la saison. (MT, p. 108-109)

Entre l'histoire effacée d'une autre et l'histoire personnelle, entre l'événement extérieur et le séisme intime, le passé et le présent, un corps et un autre corps, Ouellette-Michalska organise une circulation inédite produisant une écriture qui vient enrayer les dispositifs habituels de la transmission du discours historique patriarcal en instaurant une fluidité de l'expérience, une porosité des êtres et des temporalités, conduisant à la revendication d'une manière éminemment féminine de mettre en récit - et en fiction - l'histoire.

Cette histoire des oublié(e)s, de figures non héroïques plongées dans les circonstances ordinaires, me semble bien plus typique de la prose narrative québécoise contemporaine que les récits qui, comme la Constellation du lynx ou le Bateau d'Hitler - ou même Volkswagen

\footnotetext{
15 "Monsieur B" est la transposition du ministre Raymond Barre, qui, dans les années 1980, à l'occasion d'un voyage au Québec, visita la Maison Trestler. C'est en tombant sur le compte rendu de cette visite que la narratrice s'est intéressée à cette propriété et à son histoire.
} 
Blues qui, s'il s'attache à l'épopée grandiose de la conquête de l'Ouest, le fait toutefois du point de vue des Canadiens français, qui seront bientôt assimilés, et des Amérindiens -, entendent composer d'une manière ou d'une autre avec l'histoire majeure. En cela, cette littérature n'est pas étrangère à la mouvance du contemporain qui, comme le rappelle Jean-François Hamel à la suite de Giorgio Agamben, se range sous le paradigme de l'oubli: "Une telle mémoire de l'oubli, précise Hamel, ne concerne pas un contenu mémoriel à préserver, mais l'oubli lui-même, qu'il faudrait rappeler à la mémoire" (2006, p. 226). Dans le contexte québécois, cet oubli, c'est avant tout celui du bagage mémoriel transmis par les institutions de proximité, au premier rang desquelles la famille.

Le roman de Rachel Leclerc, la Patience des fantômes ${ }^{16}$, déploie précisément cette mémoire familiale problématique. Ici encore, comme chez OuelletteMichalska, la quatrième de couverture nous prévient qu'il s'agit de "beaucoup plus qu'une grande saga familiale sur cinq générations" ( $P F, 4^{\mathrm{e}}$ de couv.). Roman en sept parties elles-mêmes subdivisées en chapitres tournant autour d'un personnage ("Norma, 1965", "Marie, 1951", etc.) ou d'une circonstance ("les Noisettes, le naufrage, 1922", "la Réparation, 2008", etc.), la Patience des fantômes fait alterner les séquences narrées par Richard, écrivain issu de la troisième génération des Levasseur, et les séquences en narration omnisciente, ce qui permet d'éclairer diversement l'histoire de la famille, depuis le fondateur de la lignée, Joseph-Joachim, orphelin né dans la misère en Gaspésie et mort en homme d'affaires prospère dans une suite du Ritz à Montréal, jusqu'au petit Joseph qui, cinq générations plus tard, sera bientôt orphelin à son tour et recueilli par Richard. Relatée dans le désordre, cette histoire est celle d'une lignée qui d'emblée s'élève très haut dans l'échelle sociale grâce à l'intelligence et à l'énergie du patriarche pour aussitôt décliner, dès la deuxième génération. Cela dit, c'est surtout le récit de l'ascension de la génération fondatrice qui permet de croiser l'histoire familiale et l'histoire d'une région, la Gaspésie, qui se caractérise par une succession de dépossessions et d'humiliations. On voit bien, dans les lignes qui suivent, de quel ressentiment est nourrie l'ambition de Joseph-Joachim:

Il aurait l'or et il changerait à jamais le destin de ce pays de pêcheurs soumis et endettés pour des générations, il pourrait mourir, il aurait transformé en belles élégantes toutes ces femmes de la côte et aurait vengé ces hommes trop pieux, bien trop naïfs pour botter le derrière d'une poignée d'Anglais venus de Jersey pour les exploiter et les assommer avec des lois idiotes, les enchaîner à des bateaux qui ne leur appartenaient même plus. $(P F$, p. 138)
Pour sa part, le récit de la déchéance, qui repasse par toute la lignée, s'attache à déterminer de quelle nature est le fatum qui pèse sur la famille. Joseph-Joachim aurait-il à se reprocher d'avoir attiré le mauvais sort sur sa descendance? C'est l'hypothèse d'Émilie, la filleule de Richard et la mère du petit Joseph. Et pourquoi Jérôme, le seul fils survivant de Joseph-Joachim, a-t-il sombré dans l'alcoolisme et la violence au point d'anéantir sa famille? "Quelles erreurs [Joseph-]Joachim et Marie ontil donc commises, demande la femme de Jérôme, pour que les ombres t'effraient à ce point, pour qu'ensemble nous n'ayons à léguer à nos enfants que notre déchéance, notre immaturité, et pour qu'eux-mêmes soient bientôt condamnés à errer sur les côtes comme de pauvres malades" ( $P F$, p. 53-54)?

C'est bien sûr l'écrivain - lui qui ne croit pas à la malédiction mais au simple enchaînement des circonstances $^{17}$ - qui se chargera de retracer l'histoire de la famille. Pour quelle raison? Parce que "les vivants, dit-il, sont cruels envers eux-mêmes lorsqu'ils tournent le dos à leurs ancêtres, à une histoire et à une fin qui sont également les leurs" ( $P F$, p. 28). Cette dévotion à la mémoire familiale n'exclut pas la trahison, tant s'en faut; des fantômes vengeurs viennent ainsi rappeler à Richard qu'“on n'est pas autorisé, une fois qu' on est sorti $\mathrm{du}$ terrier, à revenir rôder autour pour remuer ce qui se trouve au fond" ( $P F$, p. 60-61). Mais il importe néanmoins de transmettre le récit des siens, non pas pour s'y agripper à jamais mais au contraire, semble indiquer l'auteure, pour s'en libérer. L'or dont il était question dans la citation ci-haut $^{18}$, cet or que Joseph-Joachim avait trouvé sur sa concession gaspésienne, qu'il ne pourra extraire avant de mourir et qu'il lèguera à ses descendants, cet or quasi mythique - il est là, mais personne n'a le pouvoir de s'en saisir - représente la tentation qui ronge cette famille, qui en matérialise la tragédie intime, qui en scelle le destin exceptionnel et l'impuissance congénitale: il faudra donc s'en départir comme d'un héritage importun, le liquider, ce dont se charge finalement Richard, qui note à ce propos:

Nous avons maintenant le choix: nous léguons à Joseph le cancer de notre mémoire et lui offrons un avenir plein de soucis, de vains espoirs financiers et de tractations stériles, ou nous ne le faisons pas.

\footnotetext{
${ }^{16}$ Dorénavant, les références à cet ouvrage seront appelées par le sigle $P F$ et le folio, entre parenthèses.

17 "Ils n'ont été victimes que d'eux-mêmes et de leur époque" (Leclerc, 2011, p. 12), affirme Richard dès le début du roman.

${ }^{18}$ Cet or dont Joseph-Joachim parle avec les accents rimbaldiens de "Mauvais Sang” (Une saison en enfer, 1873) - "J'aurais l'or" (PF, p. 137), "Au moins l'or [...], j'aurais trouvé au moins l'or de cette péninsule maudite" ( $P F$, p. 135) -, on en décèle sans peine les accents symboliques, qui se retrouvent dans le motif de la montre en or léguée par le patriarche, récupérée par Richard qui la fait remettre en état de marche avant d'aller l'enfouir dans la forêt, à la fin du roman, en compagnie de Joseph.
} 
Il faut vendre absolument [la concession], non pour nous mettre trois millions de dollars dans la poche comme nous allons le faire, mais pour extraire de nous, définitivement, ce qui nous ronge le cœur et la tête. D'ailleurs, peut-être aujourd'hui s'agit-il moins de récolter l'argent du rêve de grand-père que de fermer son tombeau une fois pour toutes. (PF, p. 248)

C'est là aussi l'action de l'écriture, tant romanesque qu'historienne: servir de "rite d'enterrement" afin $\mathrm{d}^{\prime \prime c}$ exorcise[r] la mort en l'introduisant dans le discours" (DE CERTEAU [1975] 2002, p. 139).

\section{Ces histoires lointaines ...: le Ciel de Bay City}

Dans le programme de la saison 2012-2013 du Théâtre d'Aujourd'hui, Guillaume Corbeil, l'auteur d'une pièce intitulée le Mécanicien, exprime son malaise devant l'affluence des touristes sur les lieux d'une mémoire douloureuse, à Auschwitz, à Ground Zero ou sur les ruines du Mur de Berlin; il en vient ensuite à ce constat:

C'est peut-être une forme de pudeur qui me gêne, comme s'il s'agissait d'une pornographie de la douleur. Si l'objet du peep-show est connu, quel est celui du spectacle de la souffrance? Quel vide cherchonsnous à combler en consommant les plaies des autres? C'est cette fascination propre à notre société épargnée - Wajdi Mouawad a même parlé de 'pays monstrueusement en paix' - que j'ai voulu questionner avec l'écriture du Mécanicien. (CORBEIL, 2012, n. p.)

Ce sentiment de malaise et de gêne, je l'ai moi-même ressenti à la lecture de textes québécois contemporains qui s'approprient les grandes catastrophes de l'histoire pour en traiter d'une manière souvent assez sommaire et extérieure - qu'il s'agisse de la Shoah ${ }^{19}$ ou du goulag ${ }^{20}$, de la guerre civile en ex-Yougoslavie ${ }^{21}$ ou en Afghanistan ${ }^{22}$. $\mathrm{Au}$ delà du jugement que l'on peut porter sur la qualité littéraire ou sur la sincérité de ces entreprises, force est pourtant de constater que l'histoire lointaine a fasciné plusieurs écrivains actuels, dont Catherine Mavrikakis qui, avec le Ciel de Bay City ${ }^{23}$, s'est taillé un impressionnant succès. Il faut dire que la Shoah se situe aujourd'hui sur le seuil de la mémoire et de la postmémoire ${ }^{24}$, d'une mémoire transmise, consciemment ou non, par la parole ou par le silence, à la deuxième et à la troisième générations, mais qui risque néanmoins de basculer dans l'histoire, c'est-à-dire de quitter la sphère immédiatement affective pour devenir un objet d'étude sans rapport direct avec l'expérience ${ }^{25}$. De telle sorte qu'on pourrait affirmer que, d'une certaine façon, nous sommes tous étrangers à Auschwitz, condamnés à conjuguer histoire, mémoire indirecte et imaginaire, et que l'ouvrage de Mavrikakis, sous ce rapport, est donc aussi légitime que n'importe quel texte écrit par les descendants de plus en plus lointains des victimes.

L'histoire du Ciel de Bay City se situe résolument à l'époque contemporaine, aux États-Unis et non au Québec - dont il n'est nullement question. La narratrice, Amy Duchesnay, écrit en 2008, près de trente ans après avoir mis le feu au bungalow de sa tante et ainsi anéanti toute sa famille immédiate. Elle se souvient de son enfance et de sa jeunesse, de la vie morne sous le ciel mauve de Bay City, cauchemar industriel du Michigan, entre le K-Mart et les terrains vagues. Elle est obsédée par le miasme toxique qui plane au-dessus de la ville, mélange de pollution industrielle, des fumées de l'incendie du bungalow et des fumées grises d'Auschwitz où ont péri la plupart des membres de sa famille, dont ses grands-parents Georges Rosenberg et Elsa Rozenweig, spectres revenus hanter le sous-sol de la demeure familiale. Car Amy est issue d'une lignée juive qui a connu la quasi extermination et qui doit sa survie à la conversion au catholicisme des sœurs Duchesnay et à leur adoption par un couple de Normands. Les deux sœurs, Denise et Babette, ont ensuite gagné l'Amérique, terre de promesses non tenues et d'amnésie pour la première, la mère d'Amy, et lieu du souvenir et de la déréliction pour la seconde. Prise entre ces deux attitudes, Amy, en proie à des cauchemars où lui apparaissent entre autres les camps de la mort, se rallie sciemment à la destinée de ses ancêtres, sans cependant trop savoir d'abord de quoi il retourne, l'histoire ne lui parvenant en effet que murmurée et par bribes. Engluée dans une vie américaine banlieusarde qu'elle juge détestable, elle est toute révolte et toute colère, à la fois désireuse de savoir et honteuse d'être née dans ce cocon

\footnotetext{
19 Voir entre autres la Constellation du Cygne de Yolande Villemaire (1985).

20 Voir Perrine Leblanc, l'Homme blanc (2010).

21 Voir David Homel, l'Analyste (2003).

${ }^{22}$ Crise en Yougoslavie, en Afghanistan ou ailleurs: ces événements peuplent les proses et les poèmes de maints écrivains qui s'en servent comme toile de fond pour dire l'horreur du monde moderne.

23 Dorénavant, les références à cet ouvrage seront appelées par le sigle $C B C$ et le folio, entre parenthèses.

${ }^{24}$ Définie par Régine Robin comme la "transmission de traumatismes de la guerre ou du génocide par ceux qui n'ont pas connu la guerre ou qui étaient trop jeunes pour comprendre la gravité des événements" (2003, p. 322). Voir aussi Hirsch (1997).

25 Selon Paul Ricœur, la conception du rapport entre mémoire et histoire chez Maurice Halbwachs "décrit ainsi une courbe: de l'histoire scolaire, extérieure à la mémoire de l'enfant, on s'est élevé à une mémoire historique qui, idéalement, se fond dans la mémoire collective qu'en échange elle agrandit, et l'on débouche in fine sur une histoire universelle qui s'intéresse aux différences d'époque et résorbe les différences de mentalité sous un regard porté de nulle part. L'histoire, ainsi reconsidérée, mérite-t-elle encore le nom de 'mémoire historique'? Mémoire et histoire ne sont-elles pas condamnées à une cohabitation forcée ?” (2000, p. 517). Pour sa part, Hartog prend acte du fait que, postérieurement aux massacres en série de la seconde moitié du XX $X^{\mathrm{e}}$ siècle, "dans le duel entre la mémoire et l'histoire, on a rapidement donné l'avantage à la première" (2003, p. 17).
} 
de laideur et de banalité, consciente de l'impossibilité de vivre normalement après Auschwitz et Treblinka et surtout après l'holocauste qu'elle-même a ordonné le 4 juillet 1979, quand, le jour même de son dix-huitième anniversaire, elle a déclenché le grand incendie ${ }^{26}$. Mystérieusement rescapée alors qu'elle voulait mourir avec le reste de sa famille, elle devient d'une certaine façon, comme Denise et Babette, inapte à témoigner: elle joint le rang de ceux qui ne peuvent parler que pour les victimes - pour leur compte et à leur place (AGAMBEN, [1998] 2003, p. 131). Or, malgré les flammes et les cendres, les morts ne meurent pas et le passé ne passe pas:

Les morts continuent leur existence. Et c'est bien là toute la tragédie des vivants, ne pas pouvoir vivre dans l'ignorance de ceux qui sont venus avant eux. C'est bien là mon terrible fardeau que d'être née de ceux qui ne sont plus et de ne rien pouvoir faire pour eux. $(C B C$, p. 52)

Impossible également d'échapper au poids du passé même si l'histoire n'est vécue que par procuration:

Je suis hantée par une histoire que je n'ai pas tout à fait vécue. Et les âmes des Juifs morts se mêlent dans mon esprit à celles des Indiens d'Amérique exterminés ici et là, sur cette terre. Ils sont tous là présents en moi, parce que l'Amérique, du Michigan au NouveauMexique, c'est cela. ( $C B C$, p. 53)

Les spectres ont beau être confinés dans les sous-sols de la mémoire, ils sont bien là, muets mais insistants. La révolte d'Amy, son désir d'abjection $(C B C$, p. 131), sa soif de "porter sur [elle] toute l'horreur du passé" $(C B C$, p. 213), viennent précisément de ce silence imposé qu'elle a entrepris de rompre en terrorisant sa tante Babette pour la forcer à parler. Les cauchemars, les hantises, les visions du ciel de Bay City enfumé par la crémation des morts s'accompagnent toutefois de certaines satisfactions amères, celles de la lucidité, de la négation de l'oubli mortifère, du consentement aussi à la condition de morte-vivante, la seule possible pour Amy à cause de sa position dans la chaîne des générations. Plus tard, pour sa fille nommée Heaven, la narratrice, faute d'être parvenue à anéantir l'histoire ${ }^{27}$, tentera à son tour d'ériger un rempart contre ses déferlements $(C B C$, p. 284); mais bien que Heaven se situe du côté du savoir et du bonheur,

\footnotetext{
${ }^{26}$ D'après ces dates (18 ans en 1979, donc naissance en 1961), la narratrice a exactement l'âge de l'auteure, et comme elle elle est née dans le Midwest (Mavrikakis à Chicago). La coïncidence ne doit certainement pas conduire à présupposer un substrat autobiographique; mais elle incite néanmoins à superposer la voix de l'auteure et celle de la narratrice, ce qui a pour résultat d'ancrer la fiction dans le réel d'une voix, d'une énonciation.

${ }^{27}$ Une histoire qui, à son insu, la poursuit jusqu'au Michigan: dans un livre intitulé Eternal Treblinka offert par sa fille, Amy apprend que la famille Ford était pro-nazie, qu'elle a soutenu Hitler, etc.

${ }^{28}$ Sur ce débat, voir Ricœur (2000).
}

les spectres finiront par la rejoindre dans le sous-sol où elle vit. Le roman se termine d'ailleurs sur une scène hallucinante où Amy la retrouve couchée entre les morts de la famille, Georges et Elsa, Babette, Denise et tous les autres. Reconstituée puis refoulée, l'histoire semble finalement avoir gagné la partie; elle se tient en tout cas dans cette situation paradoxale d'un passé récent "dont on s'étonne qu'il 'ne passe pas' ou dont on s'inquiète qu'il 'passe'” (HARTOG, 2003, p. 153).

Les exemples que j'ai évoqués - et il y en a d'autres, bien sûr - indiquent qu'il se trouve, dans la production littéraire actuelle au Québec, des romans de l'histoire au présent, même si c'est souvent une histoire mineure, diffuse ou encore lointaine qui hante les œuvres. Le fait que peu de romans prennent en charge les événements majeurs de l'histoire nationale ne veut pas dire que le passé y est minoré, loin de là - encore que le caractère insatisfaisant des rares œuvres qui conjoignent circonstances de la grande histoire et action contemporaine incite à se demander si le moment viendra bientôt où s'écrira le roman capital sur la Révolution tranquille, ou sur la crise de la conscription, ou sur les tentatives répétées de destruction de la francophonie canadienne, qui relierait ces expériences à des enjeux mémoriels, identitaires ou éthiques actuels.

Cela dit, ici comme ailleurs, les écrivains ont à composer avec l'injonction, qui vient de tous côtés, de se souvenir et de perpétuer la mémoire collective, fût-ce à travers une mémoire privée; ici aussi, ils doivent transiger avec le discours contemporain sur la postmémoire et la posthistoire - l'histoire soi-disant arrêtée après la chute du Mur de Berlin et du communisme -, de même qu'avec tout ce qui relève du devoir de mémoire, ou qui concerne le poids du passé dans le présent, l'instrumentalisation du passé dans le présent, le présent omniprésent, le passé qui passe ou ne passe pas, l'insoutenable légèreté du présent en regard du passé, et ainsi de suite.

Par ailleurs, la question des frontières entre narration fictive et narration historique, soulevée en particulier par Hamelin et abordée dans une perspective synthétique par un philosophe comme Paul Ricœur, ne se pose pas moins pour les écrivains que pour les historiens. Alors que les représentants de l'École des Annales, par exemple, ont pu se réclamer d'une histoire anti-narrativiste qui coupait court aux dangers que le récit faisait courir à l'objectivité scientifique, on a vu d'autres historiens, narrativistes ceux-là, pour qui le récit constituait en lui-même un mode d'explication et un cadre d'intelligibilité28. Quant aux romanciers, il s'en trouve plusieurs, comme François Bon en France ou Louis Hamelin au Québec, qui ont radicalement ou partiellement renoncé à la narration pour laisser parler le document brut, exhiber l'archive (vraie ou 
trafiquée, peu importe), alors que d'autres, telles Rachel Leclerc et Madeleine Ouellette-Michalska, à travers la figure de l'écrivain, et Catherine Mavrivakis, par le truchement de sa narratrice Amy, semblent s'accrocher au récit comme à l'unique façon de configurer des expériences tantôt enfouies ou mythifiées, tantôt traumatisantes. Il apparaît au total que, pour l'auteur contemporain, le problème se situe précisément dans l'élaboration d'une manière inédite de répondre aux insistantes questions que soulèvent l'histoire et la mémoire (collectives et personnelles) en ne renonçant ni aux lumières que recèlent les procédés traditionnels de la fiction, ni aux gains qu'a permis l'intégration des modèles historiques. $\mathrm{C}^{\prime}$ est ainsi que les œuvres actuelles peuvent parvenir à élaborer une rhétorique spécifique forte, qui laisse résonner les harmoniques de la conjecture, du possible, et qui, en même temps, fasse entendre le grondement des circonstances réelles telles que les circonscrivent et les conservent le témoignage oral ou la trace écrite.

\section{Références}

AGAMBEN, Giorgio. Ce qui reste d'Auschwitz. Paris: Payot \& Rivages, 2003.

BERGERON, Carl. Une œuvre fondatrice: La constellation $d u$ lynx. L'Action nationale. novembre-décembre 2010. En ligne: $<$ www.action-nationale.qc.ca/index.php?option $=$ com conte nt\&task $=$ view\&id=1110\&Itemid $=36 \&$ searchresult $=1>$ (page consultée le 2 mai 2012).

CARON, Louis. Les fils de la liberté: le Canard de bois. Montréal: Éditions du Boréal Express, 1981.

CARON, Louis. Les fils de la liberté: la Corne de brume. Montréal: Éditions du Boréal Express, 1982.

CARON, Louis. Les fils de la liberté: le Coup de poing. Montréal: Boréal, 1990.

CELLARD, Karine; LAPOINTE, Martine-Emmanuelle (Dir.). Transmission et héritages de la littérature québécoise. Montréal: Presses de l'Université de Montréal, 2011.

CORBEIL, Guillaume. Le mécanicien: Ils sont le Théâtre d'Aujourd'hui [programme de la saison 2012-2013]. En ligne: $<$ http://www.theatredaujourdhui.qc.ca/mecanicien> (page consultée le 2 mai 2012).

COUSTURE, Arlette. Les filles de Caleb: le Chant du coq. Montréal: Québec/Amérique, 1985.

COUSTURE, Arlette. Les filles de Caleb: le Cri de l'oie blanche. Montréal: Québec/Amérique, 1986.

COUSTURE, Arlette. Les filles de Caleb: l'Abandon de la mésange. Outremont: Libre Expression, 2003.

DAENINCK, Didier. Meurtres pour mémoire. Paris: Gallimard, 1984.

DASPRE, André. Le roman historique et l'histoire. Revue d'histoire littéraire de la France, Paris, v. 75, n. 2-3, p. $235-$ 244, mars./juin. 1975.
DAVID, Michel. À l'ombre du clocher: les Années folles. Montréal: Hurtubise, 2006.

DAVID, Michel. À l'ombre du clocher: le Fils de Gabrielle. Montréal: Hurtubise, 2007.

DAVID, Michel. À l'ombre du clocher: les Amours interdites. Montréal: Hurtubise, 2007a.

DAVID, Michel. À l'ombre du clocher: Au rythme des saisons. Montréal: Hurtubise, 2008.

DE CERTEAU, Michel. L'écriture de l'histoire. Paris: Gallimard, [1975] 2002.

DEMANZE, Laurent. Encres orphelines: Pierre Bergounioux, Gérard Macé, Pierre Michon. Paris: José Corti, 2008.

DESBIENS, Marie-Frédérique. Le roman historique: (r)évolution d'un genre. Québec français, Sainte-Foy, n. 140, p. 26-29, hiver 2006.

DION, Robert. Let's talk english here: les représentations de l'anglais dans Copies conformes et dans Volkswagen Blues. Une distance critique. Québec: Éditions Nota bene, 2011. p. 43-65.

DUMONT, Micheline. Romans historiques: l'histoire n'est pas une appellation contrôlée. Le Devoir, Montréal, 11 mars 2006. En ligne: <http://www.ledevoir.com/non-classe/104093/ romans-historiques-1-histoire-n-est-pas-une-appellationcontrolee $>$ (page consultée le 2 mai 2012).

FERENCZI, Thomas (Dir.). Devoir de mémoire, droit à l'oubli? Bruxelles: Éditions Complexe, 2002.

FORTIER, Dominique. Du bon usage des étoiles. Québec: Alto, 2008.

GONTARD, Marc. La maison Trestler ou le 8e jour d'Amérique, roman de Madeleine Ouellette-Michalska. Dictionnaire des œuvres littéraires du Québec: 1981-1985, Montréal: Fides, Tomo VII, p. 567-568, 2003.

HAMEL, Jean-François. Revenances de l'histoire: répétition, narrativité, modernité. Paris: Minuit, 2006.

HAMELIN, Louis. La constellation du lynx. Montréal: Boréal, [2010] 2012.

HARTOG, François. Régimes d'historicité: présentisme et expérience du temps. Paris: Seuil, 2003.

HÉBERT, Pierre. Histoires d'enlèvements. Voix et Images, Montréal, n. 48, p. 539-545, printemps 1991.

HIRSCH, Marianne. Family Frames: Photography Narrative and Postmemory. Cambridge: Harvard University Press, 1997.

HOMEL, David. L'analyste. Montréal/Arles; Leméac/Actes Sud, 2003.

LACHANCE, Micheline. Le roman de Julie Papineau: la Tourmente. Montréal: Québec/Amérique, 1995.

LACHANCE, Micheline. Le roman de Julie Papineau: l'Exil. Montréal: Québec/Amérique, 1998.

LACHANCE, Micheline. Lady Cartier. Montréal: Québec/ Amérique, 2004.

LAPIERRE, Michel. Louis Hamelin et les étoiles d'Octobre. Le Devoir, Montréal, 25 septembre 2010. En ligne: <http:// www.ledevoir.com/culture/livres/296859/louis-hamelin-et-lesetoiles-d-octobre $>$ (page consultée le 2 mai 2012). 
LEBLANC, Perrine. L'homme Blanc. Montréal: Le Quartanier, 2010.

LECLERC, Rachel. La patience des fantômes. Montréal: Boréal, 2011.

MAGINI, Roger. Revoir Nevers. Montréal: Éditions de la Pleine Lune, 2006.

MAUVIGNIER, Laurent. Des hommes. Paris: Minuit, 2009.

MAVRIKAKIS, Catherine. Le ciel de Bay City. Montréal: Héliotrope, [2008] 2011.

MERCIER, Noémi. La bombe Hamelin [Entretien avec Louis Hamelin]. L'Actualité, Montréal, 29 septembre 2010. En ligne: $<$ http://www.lactualite.com/culture/la-bombe-hamelin> (page consultée le 2 mai 2012).

OUELLETTE-MICHALSKA, Madeleine. La maison Trestler ou le 8e jour d'Amérique. Montréal: Québec/Amérique, 1984.

PATERSON, Janet M. Moments postmodernes dans le roman québécois. Ottawa: Presses de l'Université d'Ottawa, [1990] 1993.

PIÉGAY-GROS, Nathalie. Le futur antérieur de l'archive. Rimouski: Tangence Éditeur, 2012.

POULIN, Jacques. Volkswagen blues. Montréal: Québec/ Amérique, 1984.

REITSMA-LA BRUJEERE, Cora. Passé et présent dans "Les Géorgiques” de Claude Simon. Amsterdam: Rodopi, 1992.
RICEEUR, Paul. La mémoire, l'histoire, l'oubli. Paris: Seuil, 2000 .

ROBIN, Régine. La mémoire saturée. Paris, Stock, 2003.

ROBITAILLE, Antoine. Les théories du complot-1. Le Devoir, Montréal, 30 septembre 2010. En ligne: <http://www.ledevoir. com/non-classe/297189/les-theories-du-complot-1> (page consultée le 2 mai 2012).

ROLIN, Olivier. Tigre en papier. Paris: Seuil, 2002.

RUBINO, Gianfranco (Dir.). Présences du passé dans le roman français contemporain. Rome: Bulzoni, 2007.

SIMON, Claude. Les Géorgiques. Paris: Minuit, 1981.

SMART, Patricia. Écrire dans la maison du père: l'émergence du féminin dans la tradition littéraire du Québec. Montréal: Québec/Amérique, 1988.

TURGEON, Pierre. Le bateau d'Hitler. Montréal: Boréal, 1988

TURGEON, Pierre. Un dernier blues pour Octobre. Montréal: Libre Expression, 1990.

VILLEMAIRE, Yolande. La constellation du Cygne. Montréal: Éditions de la Pleine Lune, 1985.

Recebido: 18 de setembro de 2014 Aprovado: 20 de dezembro de 2014 Contato: dion.robert@uqam.ca 